\title{
IMBEDDING INFINITELY DISTRIBUTIVE LAT- TICES COMPLETELY ISOMORPHICALLY INTO BOOLEAN ALGEBRAS
}

\author{
NENOSUKE FUNAYAMA
}

Introduction. It is well known that any distributive lattice can be imbedded in a Boolean algebra ([1], [2], [4] and others). This imbedding is in general only finitely isomorphic in the sense that the imbedding preserves finite sums (supremums) and finite products (infimums) (but not necessarily infinite ones). Indeed, in order to be able to be imbedded into a Boolean algebra completely isomorphically (i.e. preserving every supremum and infimum) a distributive lattice $L$ must satisfy the infinite distributive law, as the infinite distributivity holds in Boolean algebras. The main purpose of this paper is to prove that the converse is also true, that is, any infinitely distributive lattice can be imbedded completely isomorphically in a Boolean algebra (Theorem 6). Since we show, on the other hand, that any relatively complemented distributive lattice is infinitely distributive (Theorem 2), Theorem 6 implies that every relatively complemented distributive lattice can be imbedded completely iso. morphically in a Boolean algebra (Theorem 4).

The importance of completely isomorphic imbedding is pointed out, as an example, in $\S 5$. Thus, theorems on higher degree of distributivity in Boolean algebras are generalized to those in relatively complemented distributive lattices. The theory of higher degree of distributivity in Boolean algebras has been discussed by several authors ([6], [7] and others). To generalize the theory to a relatively complemented distributive lattice $L$, we first imbed $L$ into a Boolean algebra $M$ completely isomorphically in such a manner that a property $P$ in $L$ will be preserved in $M$. If the property $P$ implies a property $Q$ in $M$, then $P$ implies $Q$ in $L$. By this method we can prove, for example, that any $(\alpha, 2)$-distributive relatively complemented lattice is $(\alpha, \alpha)$-distributive (Theorem 7).

Received October 3, 1958 
1. Preliminaries. In this section some properties of distributive lattices and of infinitely distributive lattices are stated within the scope of the necessity in this paper. A lattice $L$ is said to be infinitely distributive if the following condition is satisfied:

(I) If $a=\Sigma a_{i}\left(\Pi a_{i}\right)$, then $\Sigma b a_{i}\left(\Pi\left(b+a_{i}\right)\right)$ exists and is equal to $b a(b+a)$.

Here and hereafter $a=\Sigma a_{i}\left(\Pi a_{i}\right)$ means that $a$ is the supremum (infimum) of the set $\left\{a_{i}\right\}$. The infinite distributivity does not imply the completeness of $L$.

A mapping $\theta$ of a lattice $L$ into a lattice $L^{\prime}$ is called a completely isomorphic imbedding of $L$ into $L^{\prime}$ if the following conditions are satisfied:

(II) $a=\Sigma a_{i}\left(\Pi a_{i}\right)$ implies $\theta(a)=\Sigma \theta\left(a_{i}\right)\left(\Pi \theta\left(a_{i}\right)\right)$,

(III) $\theta(a)=\Sigma \theta\left(a_{i}\right)\left(\Pi \theta\left(a_{i}\right)\right)$ implies $a=\Sigma a_{i}\left(\Pi a_{i}\right)$.

Lеммa 1. Let $L$ be an infinitely distributive lattice and let $\Gamma: p_{1}<p_{2}<$ $\cdots<p_{n}$ be a chain in $L$. Let $L_{\Gamma}$ be the direct product of $n+1$ quotients $p_{1} / 0, p_{2} / p_{1}, \ldots, p_{n} / p_{n-1}, 1 / p_{n}$. (When $L$ lacks $0 p_{1} / 0$ means the set of elements $x$ of $L$ such that $x \leqq p_{1}$, and similarly for $1 / p_{n}$.) Let $\theta$ be the mapping of $L$ into $L_{\Gamma}$ such that $\theta(x)=\left(\ldots, \theta_{i}(x), \ldots\right)$, where $\theta_{i}(x)=p_{i}\left(x+p_{i-1}\right)$. Then $\theta$ is a completely isomorphic imbedding of $L$ into $L_{\Gamma}$.

Proof. Proof is based on the induction on the length $n$ of the chain $\Gamma$. Suppose $n=1$ and let $p_{1}=p$, then $\theta_{1}(x)=x p$ and $\theta_{2}(x)=x+p$. By the infinite distributivity of $L$ we can easily prove that $a=\Sigma a_{i}\left(\not a_{i}\right)$ in $L$ implies $\theta(a)$ $=\Sigma \theta\left(a_{i}\right)\left(\Pi \theta\left(a_{i}\right)\right)$ in $L_{\Gamma}$. For the proof of the converse implication we first show that $\theta(x) \geqq \theta(y)$ implies $x \geqq y$. Otherwise, i.e., if $x \geqq y$, then $z=x y<y$. $\theta(x) \geqq \theta(y)$ implies that $p+x \geqq p+y$ and $p x \geqq p y$, and then, since $L$ is distributive, $p+z=p+x y=(p+x)(p+y)=p+y$ and $p z=p(x y)=(p x) y \geqq(p y) y$ $=p y \geqq p z$. Two equalities $p+z=p+y$ and $p z=p y$ and the inequality $z<y$ contradict to the distributivity of $L$. Now suppose that $\theta(a)=\Sigma \theta\left(a_{i}\right)$, then $\theta(a) \geqq \theta\left(a_{i}\right)$ for every $i$. Then $a \geqq a_{i}$ for every $i$, and so $a$ is an upper bound of $\left\{a_{i}\right\}$. Suppose that $y$ is any upper bound of $\left\{a_{i}\right\}$, then $\theta(y) \geqq \Sigma \theta\left(a_{i}\right)=\theta(a)$, which implies $y \geqq a$. Thus we have $a=\Sigma a_{i}$. In the same way we can show that $\theta(a)=\Pi \theta\left(a_{i}\right)$ implies $a=\Pi a_{i}$.

Assume that the conclusion is true for chains whose lengths are less than $n$. Let $I^{\prime}$ be the subchain $p_{1}<p_{2}<\cdots<p_{n-1}$ of $I$. Then the canonical mapping $\phi$, defined in the lemma, of $L$ into $L_{\Gamma}$, is a completely isomorphic imbedding by the inductive assumption. Let $\psi$ be the mapping of $L_{\Gamma}$ into $L_{\Gamma}$. 
which is defined as the identical mapping on $p_{1} / 0 \otimes \cdots \otimes p_{n-1} / p_{n-2}$ and the canonical mapping on $1 / p_{n-1}$ (into $p_{n} / p_{n-1} \otimes 1 / p_{n}$ ). Then $\psi$ is a completely isomorphic imbedding of $L_{\Gamma}$ into $L_{\Gamma}$ by the case $n=1$. As is easily seen, we have $\theta=\psi \phi$. As the product of completely isomorphic imbeddings is a completely isomorphic imbedding, $\theta$ is a completely isomorphic imbedding of $L$ into $L_{\Gamma}$.

Although the following Lemmas 2-4 are perhaps more or less well known, we will prove them briefly for the sake of completeness. They could be proved directly without using prime ideals, but then the proofs would require tedious computations. So we use the following fact which we give here without proof: For any pair of elements $p>q$ in a distributive lattice there exists a prime ideal which contains $q$ and not containing $p$. This prime ideal is said to divide $p / q$.

Leмma 2. In a distributive lattice $L$ the following conditions for two quotients $p / q$ and $r / s$ are equivalent:

(1) $p / q$ and $r / s$ are projective,

(2) The set of all prime ideals which divide $p / q$ is equal to the set of all prime ideals which divide $r / s$,

(3) There exists a quotient $t / u$ such that $p / q$ and $t / u$, and $r / s$ and $t / u$ are transposes. Moreover we can take $u=q+s$ and $t=p+s(=q+r=p+r)$.

Proof. Clearly (1) implies (2) as the projective quotient is obtained by a series of transposed quotients. Assume (2) and put $q+s=u$ and $t=p+s$. Then clearly $p+u=t$. Suppose that $p u=q^{\prime}>q$, then there exists a prime ideal $P$ which divides $q^{\prime} / q$. $P$ divides $p / q$ and so divides $r / s$ by (2). Then $P$ includes $q$ and $s$ and then $u$ and so $q^{\prime}$, which contradicts to the assumption that $P$ divides $q^{\prime} / q$. Thus we have $p u=q$. In the same way we can show that $u r=s$ and $u+r=t$. That (3) implies (1) is clear.

Lemma 3. Let $p / q$ and $r / s$ be projective quotients in a distributive lattice L. Let $\theta: p / q \rightarrow r / s, \phi: L \rightarrow p / q$ and $\psi: L \rightarrow r / s$ be mappings such that $\theta(x)$ $=r(x+s), \phi(x)=p(x+q)$ and $\psi(x)=r(x+s)$. Then $\theta$ is an isomorphism onto, and $\theta \phi=\psi$.

Proof. Put $q+s=u$ and $p+s=t$, then by Lemma 2 we have $p+u=t$ and $r u=s$. Then $\theta(x)=r(x+s)=r(x+u)$ for $x \in p / q$, and $x \rightarrow y=x+u$ gives an 
isomorphism of $p / q$ and $t / u$, while $y \rightarrow r y=\theta(x)$ gives an isomorphism of $t / u$ and $r / s$. Thus $\theta$ gives an isomorphism of $p / q$ and $r / s$.

$$
\begin{aligned}
\theta \phi(x) & =r(p(x+q)+s)=r(p x+u)=r(p+u)(x+u) \\
& =r t(x+u)=r(x+u)=r x+r u=r x+s=\psi(x) .
\end{aligned}
$$

Lemma 4. Let $L$ be a finite dimensional (and so finite) distributive lattice. Let $\Gamma: 0<p_{1}<p_{2}<p_{3}<\cdots<p_{n}=1$ be a complete chain in L. Then in $\Gamma$ no two prime quotients are projective. Let $\Omega: 0<q_{1}<q_{2}<\cdots<q_{n}=1$ be another complete chain $L$. Then there exists a (unique) one-to-one correspondence between the set of prime quotients $\left\{p_{i} / p_{i-1}, i=1,2, \ldots, n\right\}$ and $\left\{q_{j} / q_{j-1}\right.$, $j=1,2, \ldots, n\}$, where $p_{i} / p_{i-1}$ corresponds to $q_{j} / q_{j-1}$ if and only if one of the following equivalent conditions is satisfied.

(1) $p_{i} / p_{i-1}$ is projective to $q_{j} / q_{j-1}$.

(2) Any prime ideal dividing $p_{i} / p_{i-1}$ divides $q_{j} / q_{j-1}$.

(3) $q_{j}\left(p_{i}+q_{j-1}\right)=q_{j}$ and $q_{j}\left(p_{i-1}+q_{j-1}\right)=q_{j-1}$.

(4) $p_{i}\left(q_{j}+p_{i-1}\right)=p_{i}$ and $p_{i}\left(g_{j-1}+p_{i-1}\right)=p_{i-1}$.

Proof is clear from Lemma 2. The correspondence between the sets of prime quotients in two complete chains is called the canonical correspondence.

Lemma 5. Let $D$ be a finite sublattice of a distributive lattice L. Let $\Gamma: p_{1}<p_{2}<\cdots<p_{n}$ and $\Omega: q_{1}<q_{2}<\cdots<q_{n}$ be complete chains in $D$, and let $L_{\Gamma}=p_{1} / 0 \otimes p_{2} / p_{1} \otimes \cdots \otimes 1 / p_{n}$ and $L_{2}=q_{1} / 0 \otimes q_{2} / q_{1} \otimes \cdots \otimes 1 / q_{n}$. Then $L_{\Gamma}$ and $L_{\Omega}$ are isomorphic.

Proof. By the canonical correspondence we have a (unique) one-to-one correspondence between the direct product factors in such a manner that the corresponding factors are projective, whence, by Lemma 3, isomophic.

The isomorphism between $L_{\Gamma}$ and $L_{\Omega}$ just defined is called the canonical isomorphism between $L_{\Gamma}$ and $L_{\Omega}$, and is denoted as $\theta_{\Gamma}^{\Omega}$.

By this lemma we have showed that $L_{\Gamma}$ does not depend on the choice of $\Gamma$ within isomorphism, and so may be written as $L_{D}$.

Leмma 6. If $L$ is infinitely distributive, then $L_{D}$ is infinitely distributive for any finite sublattice $D$ of $L$.

This is clear from the fact that the direct product factors in $L_{D}$ are 
infinitely distributive.

LEMMA 7. Let $D$ and $E$ be finite sublattices of an infinitely distributive lattice $L$ such that $D \subset E$. Then $L_{D}$ is completely isomorphically imbedded in $L_{\vec{r}}$.

Proof. Suppose that $L_{D}$ and $L_{E}$ are determined as $L_{\Gamma}$ and $L_{\Omega}$, where $\Gamma: p_{1}<p_{2}<\cdots<p_{n}$ and $\Omega: q_{1}<q_{2}<\cdots<q_{m}$ are complete chains respectively in $D$ and $E$. Let $\Omega^{\prime}: r_{1}<r_{2}<\cdots<r_{m}$ be a complete chain in $E$, which is a subdivision of $\Gamma$, and so suppose that $r_{1}<\cdots<r_{k(1)}=p_{1}<\cdots<r_{k(2)}$ $=p_{2}<\cdots<r_{k(n)}=p_{n}<\cdots<r_{m}$. Let $\phi_{i}: p_{i} / p_{i-1} \rightarrow r_{k(i-1)+1} / r_{k(i-1)} \otimes \cdots \otimes r_{k(i)} \mid$ $r_{k(i)-1}$ be the canonical completely isomorphic imbedding introduced in Lemma 1. $\phi_{i}$ 's give a completely isomorphic imbedding $\theta_{\Gamma}^{Q^{\prime}}$ of $L_{\Gamma}=L_{D}$ into $L_{\Omega^{\prime}}$. Then $\theta_{\Omega^{\prime}} \theta_{\Gamma}^{\Omega^{\prime}}$ gives a completely isomorphic imbedding of $L_{D}$ into $L_{E}$.

Lemma 8. $\theta_{\Omega^{\prime}}^{\Omega} \theta_{\Gamma}^{Q^{\prime}}$ does not depend on the choice of $\Omega^{\prime}$.

Proof. Let $\Omega^{\prime \prime}$ be another complete chain in $E$ which is a subdivision of $\Gamma$. Let us suppose that $s_{l} / s_{l-1}$ is a prime quotient in $\Omega^{\prime \prime}$ whose corresponding quotients in $\Omega$ and $\Omega^{\prime}$ are respectively $q_{j} / q_{j-1}$ and $r_{k} / \boldsymbol{r}_{k-1}$. Then $q_{j} / q_{j-1}$ corresponds to $r_{k} / r_{k-1}$ as the correspondence is settled by projectivity. Let $p_{i} / p_{i-1}$ be a quotient in $\Gamma$ which includes $\boldsymbol{r}_{k} / \boldsymbol{r}_{k-1}$ (and so $s_{l} / \boldsymbol{s}_{l-1}$ ) and let $x$ be any element in $L$ which lies in $p_{i} / p_{i-1}$. Then $x$ is mapped in $q_{j} / q_{j-1}$ through $\Omega^{\prime}$ on

$$
q_{j}\left(r_{k}\left(x+r_{k-1}\right)+q_{j-1}\right)=q_{j}\left(x+q_{j-1}\right)
$$

as was proved in Lemma 2 , and on the same element through $\Omega^{\prime \prime}$ too.

The following lemma follows from Lemma 8 and Lemma 2 .

Lemma 9. Let $L$ be an infinitely distributive lattice and let $D, E$ and $F$ be finite sublattices such that $D \subset E \subset F$. Then $\theta_{D}^{F}=\theta_{E}^{F} \theta_{D}^{F}$.

2. Direct limit of lattices. Let $\left\{L_{\lambda} ; \lambda \in \Lambda\right\}$ be a set of lattices indexed by a directed set $A . \quad\left\{L_{\lambda}\right\}$ is called a directed system of lattices if there is given a complete isomorphisms $\phi_{\lambda}^{u}$ of $L_{\lambda}$ into $L_{\mu}$ for every pair $\lambda \leqq \mu$ such that when $\lambda=\mu, \phi_{\lambda}^{u}$ is the identity mapping in $L_{\lambda}$ which

(*) $\phi_{i}^{\nu} \phi_{\lambda}^{\mu}=\phi_{\lambda}^{\nu}$ for any triplet $\lambda \leqq \mu \leqq \nu$.

On the set of all elements of $L_{\lambda}$ 's we define a binary relation $\sim$ by

( ) For $x_{\lambda} \in L_{\lambda}, x_{\mu} \in L_{\mu}, x_{\lambda} \sim x_{\mu}$ if and only if for some $\nu \geqq \lambda, " n, \phi_{\lambda}^{\nu}\left(x_{\lambda}\right)$ $=\phi_{\mu}^{2}\left(x_{\mu}\right)$. 
This condition, as is easily seen from the complete isomorphism of $\phi_{\lambda}^{\mu}$, is equivalent to the condition that $\phi_{\lambda}^{\nu}\left(x_{\lambda}\right)=\phi_{\mu}^{\nu}\left(x_{\mu}\right)$ for every $\nu \geqq \lambda, \mu$. $\sim$ is an equivalence relation. Denote the $\sim$-equivalence class containing $x_{\lambda}$ by $\left[x_{\lambda}\right]$. On the set of equivalence classes $\left\{\left[x_{\lambda}\right]\right\}$ we define a binary relation $\geqq$, $\left[x_{\lambda}\right] \geqq\left[x_{\mu}\right]$ if and only if for some (and equivalently for every) $\nu \geqq \lambda, \mu$, $\phi_{\lambda}^{\prime}\left(x_{\lambda}\right) \geqq \phi_{\mu}^{\nu}\left(x_{\mu}\right)$ in $L_{\nu}$. Then the set $L$ of all equivalence classes $\left[x_{\lambda}\right]$ forms a lattice by the relation $\geqq$. $L$ is called the direct limit of the directed system of lattices $\left\{L_{\lambda} ; \phi_{\lambda}^{\mu}\right\}$.

LEMMA 10. Let $L$ be the direct limit of the directed system of lattices $\left\{L_{\lambda} ; \phi_{\lambda}^{\mu}\right\}$, and let $\phi_{\lambda}$ be a mapping of $L_{\lambda}$ into $L$ such that $\phi_{\lambda}\left(x_{\lambda}\right)=\left[x_{\lambda}\right]$. Then $\phi_{\lambda}$ is a completely isomorphic imbedding of $L_{\lambda}$ into $L$.

Proof. Suppose that $x=\Sigma x_{i}$ in $L_{\lambda}$, then by the definition $\phi_{\lambda}(x) \geqq \phi_{\lambda}\left(x_{i}\right)$ for all $i$, that is, $\phi_{\lambda}(x)$ is an upper bound of $\left\{\phi_{\lambda}\left(x_{i}\right)\right\}$. Suppose that $[y] \geqq \phi_{\lambda}\left(x_{i}\right)$ for all $i$ and for some $y \in L_{\mu}$. Then for every $\nu \geqq \lambda, \mu, \phi_{\mu}^{\nu}(y) \geqq \phi_{\lambda}^{\nu}\left(x_{i}\right)$. On the other hand, as $\phi_{\lambda}^{\nu}$ is a complete isomorphism of $L_{\lambda}$ into $L_{\nu}, \phi_{\lambda}^{\nu}(x)=\Sigma \phi_{\lambda}^{\nu}\left(x_{i}\right)$. Thus $\phi_{\mu}^{\nu}(y) \geqq \phi_{\lambda}^{\nu}(x)$, or equivalently, $[y] \geqq \phi_{\lambda}(x)$. Thus we have $\phi_{\lambda}(x)=\Sigma \phi_{\lambda}\left(x_{i}\right)$ in $L$.

That $\phi_{\lambda}(x)=\Sigma \phi_{\lambda}\left(x_{i}\right)$ for $x$ and $x_{i}$ 's in $L_{\lambda}$ implies $x=\Sigma x_{i}$ in $L_{\lambda}$ is easily shown.

LEммA 11. $\phi_{\mu} \phi_{\lambda}^{\mu}=\phi_{\lambda}$ for $\lambda \leqq \mu$.

THEOREM 1. Let $L$ be the direct limit of the directed system of lattices $\left\{L_{\lambda} ; \phi_{\lambda}^{\mu}\right\}$. Then $L$ is modular or distributive if and only if all the $L_{\lambda}$ 's are modular or distributive. $L$ is relatively complemented if and only if for any three elements $x<y<z$ in $L_{\lambda}$ ( $\lambda$ is arbitrary) there exists $\nu \geqq \lambda$ such that $\phi_{\lambda}^{\prime}(y)$ has a relative complement relative to $\phi_{\lambda}^{\prime \prime}(x)$ and $\phi_{\lambda}^{\nu}(z)$ in $L_{\nu} . \quad L$ has the unit and zero if and only if there exists some $\lambda_{0}$ such that for every $\nu \geqq \lambda_{0}, L_{\nu}$ has the unit and zero and $\phi_{\lambda_{0}}^{\nu}$ maps the unit and zero in $L_{\lambda_{0}}$ on the unit and zero in $L_{\nu}$. In this case $L$ is complemented if and only if for every $\mu$ and any $x \in L_{\mu}$ there exists $\nu \geqq \mu$ such that $\phi_{\mu}^{\nu}(x)$ has a complement in $L_{\nu}$.

3. Infinite distributivity of relatively complemented distributive lattices. It is well known that any Boolean algebra is infinitely distributive. We have the following generalization of this theorem. 
THEOREM 2. Every relatively complemented distributive lattice is infinitely distributive.

Proof. Let $L$ be a relatively complemented distributive lattice, and let us suppose $x=\Sigma x_{\lambda}$. Fix any element $x_{0}$ in $\left\{x_{\lambda}\right\}$. Then $\left\{x_{\lambda}+x_{0}\right\}$ are in the quotient lattice $x+y / y x_{0}$ for any element $y$ in $L$. As $x+y / y x_{0}$ is a Booean algebra it is infinitely distributive. Then $x=\Sigma\left(x_{\lambda}+x_{0}\right)$ in $x+y / y x_{0}$ implies that $y x=\Sigma y\left(x_{\lambda}+x_{0}\right)=\Sigma\left(y x_{\lambda}+y x_{0}\right)=\Sigma y x_{\lambda}$.

Similary $x=\Pi x_{\lambda}$ implies $y+x=\Pi\left(y+x_{\lambda}\right)$ for any element $y \in L$.

\section{Adjoining 1 and 0 for infintely distributive lattices}

Theorem 3. Any infinitely distributive lattice can be imbedded completely isomorphically into an infinitely distributive lattice with unit (1) and zero (0).

Proof. Let $L$ be an infinitely distributive lattice, and let $L$ be the direct product of all quotient lattices in $L$. Clearly $\bar{L}$ has 1 and 0 and is infinitely distributive as the same is the case with every direct product factor. Let $\theta_{p / q}: L \rightarrow p / q$ be such that $\theta_{p ! q}(x)=p(x+q)$, and let $\theta: L \rightarrow L$ be such that the $p / q$-component of $\theta(x)$ is $\theta_{p / q}(x)$. We now show that $\theta$ is a completely isomorphic imbedding of $L$ into $L$. Suppose that $x=\Sigma x_{\lambda}$ in $L$, then for any $p / q$ we have $\theta_{p / q}(x)=p(x+q)=p\left(\left(\Sigma x_{\lambda}\right)+q\right)=p\left(\Sigma\left(x_{\lambda}+q\right)\right)=\Sigma p\left(x_{\lambda}+q\right)=\Sigma \theta_{p / q}\left(x_{\lambda}\right)$, that is, $\theta(x)=\Sigma \theta\left(x_{\lambda}\right)$. Likewise we can show that $x=\Pi x_{\lambda}$ implies $\theta(x)=I r \theta\left(x_{\lambda}\right)$.

For the proof of the converse implication we first show that $\theta(x) \geqq \theta(y)$ implies $x \geqq y . \quad \theta(x) \geqq \theta(y)$ implies $p(x+q) \geqq p(y+q)$ for any quotient $p / q$. If $x \neq y$, then $x+y>x y$. Put $p=x+y$ and $q=x y$ in $p(x+q) \geqq p(y+q)$, then we have $x \geqq y$. Thus we have that $\theta(x) \geqq \theta(y)$ implies $x \geqq y$ or $x=y$, that is, $x \geqq y$. Now suppose that $\theta(x)=\Sigma \theta\left(x_{\lambda}\right)$. Then $\theta(x) \geqq \theta\left(x_{\lambda}\right)$ for every $\lambda$, and then $x \geqq x_{\lambda}$ for every $\lambda$, that is, $x$ is an upper bound of $\left\{x_{\lambda}\right\}$. Let $y$ be any upper bound of $\left\{x_{\lambda}\right\}$, then $y \geqq x_{\lambda}$, which implies $\theta(y) \geqq \theta\left(x_{\lambda}\right)$ for every $\lambda$. Then $\theta(y) \geqq \Sigma \theta\left(x_{\curlywedge}\right)=\theta(x)$. This implies $y \geqq x$. Thus $x$ is the least upper bound of $\left\{x_{\lambda}\right\}$. Similary we can show that $\theta(x)=I I \theta\left(x_{\lambda}\right)$ implies $x=I I x_{\lambda}$.

THEOREM 4. Any relatively complemented distributive lattive $L$ can be imbedded completely isomorphically in a Boolean algebra.

Proof. $L$ is infinitely distributive by Theorem 2 . Let $\bar{L}$ be the direct product of all quotients in $L$ as introduced in Theorem 3. Then $L$ is a Boolean 
algebra as all the direct product factors are. By Theorem 3 we have the proof.

Remark. If $L$ is a distributive lattice and not infinitely distributive. Then $L$ is imbedded in $L$ only finitely isomorphically, which implies that any distributive lattice can be imbedded in a Boolean algebra. Even in this case if $x=\Sigma x_{\lambda}$ is an infinitely distributive sum, i.e., for any element $y$ in $L, \Sigma y x_{\lambda}$ (exists and) $=y x$ implies $\theta(x)=\Sigma \theta\left(x_{\lambda}\right)$.

\section{Imbedding infinitely distributive lattices into Boolean algebras}

By Theorem 4 in order to imbed an infinitely distributive lattice $L$ completely isomorphically into a Boolean algebra it is sufficient to imbed $L$ completely isomorphically into a relatively complemented distributive lattice. Moreover, by Theorem 3, we can assume that $L$ has 1 and 0 . Therefore from now on let us suppose that $L$ is an infinitely distributive lattice with 1 and 0 . Let $\Delta=\{D, E, F, \ldots\}$ be the set of all finite sublattices of $L$ which contain 1 and $0 . \quad \Delta$ is a directed set under set inclusion since the sublattice of $L$ generated by a finite subset of $L$ is finite.

We will contruct a directed system of lattices begining with $L$. Let $L_{D}$ $(D \in \Delta)$ be the infinitely distributive lattice defined in $\S 1$, and let $\theta_{D}^{E}$ be the completely isomorphic imbedding of $L_{D}$ into $L_{F}$ for $D \subset E$, which was defined in Lemma 7. Then $\left\{L_{D} ; \theta_{D}^{E}\right\}$ is a directed system of lattices by Lemma 9. Let $M$ be the direct limit of this directed system of lattices. Then

\section{Lemma $12 . \quad M$ is relatively complemented.}

Proof. By Theorem 1 it is sufficient to prove that for any three elements $x<y<z$ in $L_{D}$ there exists $E>D$ such that $\theta_{D}^{E}(y)$ has a relative complement relative to $\theta_{D}^{E}(x)$ and $\theta_{D}^{F}(z)$ in $L_{E}$. Let us suppose that $L_{D}$ is defined as $L_{\Gamma}=p_{1} / 0 \otimes \cdots \otimes 1 / p_{n-1}$, and that $x, y, z$ are represented respectively as $x=\left(x_{1}, \ldots, x_{n}\right), \mathrm{y}=\left(y_{1}, \ldots, y_{n}\right)$ and $z=\left(z_{1}, \ldots, z_{n}\right)$, where $x_{i}, y_{i}, z_{i} \in p_{i} / p_{i-1}$ for $i=1, \ldots, n$. Let $E$ be the sublattice of $L$ generated by $D \cup\left\{x_{1}, \ldots, x_{n}\right.$, $\left.y_{1}, \ldots, y_{n}, \ldots, z_{1}, \ldots, z_{n}\right\rangle$. Then $E \in \Delta$, and since $x<y<z$ we have a chain in $E, \Omega^{\prime}: x_{1} \leqq y_{1} \leqq z_{1} \leqq p_{1} \leqq x_{2} \leqq y_{2} \leqq z_{2} \leqq p_{2} \leqq \cdots \leqq p_{n-1} \leqq x_{n} \leqq \dot{y}_{n} \leqq z_{n}$. Let $\Omega$ be a complete chain in $E$ which is a subdivision of $\Omega^{\prime}$. In $L_{\Omega}$, which may be considered as $L_{E}, \theta_{D}^{E}(y)$ has a relative complement relative to $\theta_{D}^{E}(x)$ and $\theta_{D}^{E}(z)$. Q.E.D. 
LEMMA 13. $L$ is completely isomorphically imbedded in $M$.

Proof. $L=L_{\{0,1\}}$ is completely isomorphically imbedded in $M$ by Lemma 10.

TheOREM 5. Any infinitely distributive lattice can be imbedded completely isomorphically in a relatively complemented distributive (and hence infinitely distributive) lattice.

By Theorem 5 and 4 we have

Theorem 6. (Main Theorem) Any infinitely distributive lattice can be imbedded in a Boolean algebra completely isomorphically.

The construction of the Boolean algebra into which the given infinitely distributive lattice is imbedded indicates the following corollary.

Corollary 1. Any infinitely distributive lattice with 1 and 0 can be imbedded completely isomorphically into a Boolean algebra in such a manner that the unit and zero are mapped on the unit and zero, and complements on complements.

As the completion by cuts of a Boolean algebra is a Boolean algebra we have

Corollary 2. Any infinitely distributive lattice can be imbedded in a com plete Boolean algebra completely isomorphically.

This corollary includes the following corollary, which is a solution of the problem raised in [3].

Corollary 3. Any infinitely distributive lattice can be imbedded completely isomorphically in an infinitely distributive complete lattice.

\section{Higher distributivity in relatively complemented lattices}

Higher distributivity in Boolean algebras was discussed in [6] and others. In this section we use the same definitions and notations as in [6]. We call a lattice $L$ is $(\alpha, \beta)$-distributive, where $\alpha$ and $\beta$ are cardinal numbers, if the following conditions are satisfied: (1) Given any double sequence $a \in L^{\alpha \times \beta}$ such that if all the sums $\Sigma_{\eta<\beta} a_{\xi, \eta}$ for $\xi<\alpha$, their product $\Pi_{\xi<\alpha} \sum_{\eta_{i}<\beta} a_{\xi, \eta}$, and all the products $\Pi_{\xi<\alpha} a_{\xi, f(\xi)}$ for all $f \in \beta^{\alpha}$ exist, then the sum $\Sigma_{f \in \beta} \alpha I_{\xi<\alpha} a_{5, f(\xi)}$ also exists, and we have $\Pi_{\xi<\alpha} \Sigma_{\eta<\beta} a_{\xi, \eta}=\Sigma_{f \in \beta \alpha} \Pi_{\xi<\alpha} a_{\xi, f(\xi)}$. (2) The dual of (1). 
Notice that in [6], the dual (2) was not needed since there was discussed only Boolean algebras and in a Boolean algebra there exists duality. Using Theorem 4 a large number of theorems in [6] concerning higher distributivity in Boolean algebras are extended to those in relatively complemented lattices. As the proofs are similar we present here one of them.

THEOREM 7. Any $(\alpha, 2)$-distributive relatively complemented distributive lattice is $(\alpha, \alpha)$-distributive.

Proof. Let $L$ be an $(\alpha, 2)$-distributive relatively complemented (distributive) lattice, and let $M$ be the direct product of all quotients in $L$. Then $M$ is an $(\alpha, 2)$-distributive Boolean algebra as all the direct product factors are Boolean algebra and $(\alpha, 2)$-distributive. By Theorem $4 L$ is imbedded completely isomorphically into $M$ by a mapping $\theta$. Now suppose that for a double sequence $a \in L^{\alpha \times \alpha}, \Pi_{\xi<\alpha} \Sigma_{\eta_{i<\alpha}} a_{5, \eta}$ and all the products $\Pi_{\xi<\alpha} a_{\xi, f(\xi)}$ exist for all $f \in \alpha^{\alpha}$.

Then, since $\theta$ is a completely isomorphic imbedding, $\Pi_{5<\alpha} \Sigma_{\eta<\beta} \theta\left(a_{5, \eta}\right)$ and the products $\Pi_{\xi<\alpha} \theta\left(a_{\xi, f(\xi)}\right)$ exist for all $f \in \alpha^{\alpha}$. As $M$ is an $(\alpha, 2)$-distributive Boolean algebra it is $(\alpha, \alpha)$-distributive $([6])$. Then we have

$$
\Pi_{\xi<\alpha} \Sigma_{\eta<\alpha} \theta\left(a_{\S, \eta}\right)=\Sigma_{f \in \alpha^{\alpha}} \Pi_{\xi<\alpha} \theta\left(a_{\xi, f(\xi)}\right) .
$$

Again since $\theta$ is a completely isomorphic imbedding we have $\Pi_{\xi<\alpha} \Sigma_{\eta_{i<\alpha}} \theta\left(a_{\xi}, \eta\right)$ $=\Pi_{\xi<\alpha} \theta\left(\Sigma_{\eta_{<\alpha}} a_{\xi, \eta}\right)$, as $\Sigma_{\eta<\alpha} a_{\xi, \eta}$ exists. Also $\Pi_{\xi<\alpha} \theta\left(\Sigma_{\eta_{i}<\alpha} a_{\xi, \eta}\right)=\theta\left(\Pi_{\xi<\alpha} \Sigma_{\eta<\alpha} a_{\xi, \eta}\right)$ as $\Pi_{\xi<\alpha} \Sigma_{\eta<\alpha} a_{\xi, \eta}$ exists. For every $f \in \alpha^{\alpha}$ we have $\Pi_{\xi<\alpha} \theta\left(a_{\left.\xi, f_{1}\right)}\right)=\theta\left(\Pi_{\xi<\alpha} a_{\xi, f(\xi)}\right)$ as $\Pi_{\xi<\alpha} a_{\xi, f(\xi)}$ exists for every $f \in \alpha^{\alpha}$. Thus we have that

$$
\theta\left(\Pi_{\xi<\alpha} \Sigma_{\eta<\alpha} a_{\xi, \eta}\right)=\Sigma_{f \in \alpha^{\alpha}} \theta\left(\Pi_{\xi<\alpha} a_{\xi, f(\xi)}\right) .
$$

Again since $\theta$ is a completely isomorphic imbedding we have

$$
\Sigma_{f \in \alpha^{\alpha}} \Pi_{\xi<\alpha} a_{\xi, f(\xi)}=\Pi_{\xi<\alpha} \Sigma_{\eta<\alpha} a_{\xi, \eta} .
$$

\section{REFERENCES}

[1] G. Birkhoff, Lattice Theory, Revised edition, Amer. Math. Soc. Colloq. Publ., vol. 25 (1948).

[2] G. Birkhoff, On rings of sets, Duke Math. J., vol. 3 (1937), pp. 443-454.

[3] N. Funayama, Imbedding partly ordered sets into infinitely distributive complete lattices, Tóhoku Math. J., vol. 8 (1956), pp. 54-62.

[4] W. Peremans, Embedding of a distributive lattice into a Boolean algebra, Nederl. Akad. Wetensch. Proc. Ser. A, 60=Indag. Math., vol. 19 (1957), pp. 73-81.

[5] J. Schmidt, Die transfiniten Operationen der Ordnungstheorie, Math. Ann., vol. 133 (1957), pp. 439-449. 
[6] E. C. Smith and A. Tarski, Higher degree of distributivity and completeness in Boolean algebras, Trans. Amer. Math. Soc., vol. 84 (1957), pp. 230-257.

[7] E. C. Smith, A distributivity condition for Boolean algebras, Ann. Math., vol. 64 (1956), pp. $551-562$.

Yamagata University 